\title{
A quantile-based anthropometry synthesis technique for global user populations
}

\author{
Gopal Nadadur ${ }^{\mathrm{a}}$, Ulrich Raschke ${ }^{\mathrm{b}}$, Matthew B. Parkinson ${ }^{\mathrm{a}}$ \\ ${ }^{a}$ The Pennsylvania State University, 213 Hammond Building, University Park, PA \\ 16802, USA \\ ${ }^{b}$ Siemens PLM Software
}

\begin{abstract}
Detailed anthropometric data are valuable in making well-informed and responsible design decisions. However, such data are available only for a few user populations around the world. More widely-available information is in the form of summary statistics (e.g., means and standard deviations) and the values of body measures at certain key percentiles (e.g., $\left.5^{\text {th }}, 50^{\text {th }}, 95^{\text {th }}\right)$. Such information, while useful, is not suitable for in-depth analyses of a population's variability, since it does not allow for the consideration of correlations between different body measures, does not describe irregular distributions of body dimensions, etc. This paper presents a new methodology that utilizes values of body measures at different percentiles in synthesizing a detailed anthropometric database for a virtual population of users. The procedure is demonstrated in the context of Japanese civilian youth and U.S. military, and is shown to be simple, accurate, easy to use, and applicable across these two anthropometrically dissimilar populations. The case study shows that the virtual population is statistically equivalent to the actual target population in a number of ways. In addition to achieving statistical equivalence with the actual population's body dimensions, the method also ensures that the synthesized individuals are composed of appropriate and realistic body proportions and combinations of anthropometry.
\end{abstract}

Keywords: anthropometry, global populations, human variability

\section{Introduction}

Anthropometry (i.e., body measures) are known to be influential in determining users' preferred styles of physically interacting with products (Strasser,

Preprint submitted to International Journal of Industrial Ergonomics

April 20, 2015

(C) 2015. This manuscript version is made available under the Elsevier user license http://www.elsevier.com/open-access/userlicense/1.0/ 
1995). Information about anthropometry is vital to satisfying product performance objectives such as safety, comfort, ease of use, and visibility. This paper presents a methodology for the synthesis of detailed databases of anthropometry for "virtual populations" that are comparable to the actual target populations. The method is based on readily-available information about the required body measures for the population. The resulting databases allow for in-depth analyses of the variability of relevant body dimensions in the target population; doing so is key to developing ergonomic designs that efficiently accommodate the desired percentage of users.

\subsection{Sources of information about population anthropometry}

Anthropometric databases contain the values of body dimensions measured for a surveyed population of individuals. Such databases are available for certain user populations; examples include ANSUR (Gordon et al., 1989), which is representative of the U.S. military in the late 1980s, NHANES (U.S. Centers for Disease Control and Prevention, 2008), which provides limited data about the civilian U.S. population, and CAESAR (Blackwell et al., 2008), which is a convenience sample of North American and European populations. However, due to the resources and effort entailed in the compilation of such databases, they are few in number. Additionally, data collected from studies are often not made publicly available. A compounding complication for the designer is the uniqueness of the anthropometric characteristics of distinct populations. This uniqueness renders inaccurate the substitution of other populations' data in place of the target users' anthropometry.

Numerous studies through the years have resulted in concise statistical information about a variety of populations. This statistical information is usually in the form of summary statistics - the mean and standard deviation of different body measures - and can also include the values of these body measures at key percentiles. For example, Gite et al. (2009) provides the means and standard deviations of body measures, which are assumed to be normally distributed, of Indian farm workers. The State Bureau of Technical Supervision (1989) and Instituto Nacional de Tecnologia (1995) are sources of values of anthropometry at certain percentiles (e.g., $1^{\text {st }}, 5^{\text {th }}, 10^{\text {th }}, 50^{\text {th }}, 90^{\text {th }}$, $95^{\text {th }}$, and $99^{\text {th }}$ in the former) for Chinese and Brazilian civilians, respectively. Barroso et al. (2005) provides both summary statistics as well as percentilewise information of anthropometry for the Portuguese worker population.

Many surveys have been directed at more specific groups of users, including Algerian date palm farmers (Mokdad, 2002), Bahraini school chil- 
dren between 6 and 12 years of age (Mokdad \& Al-Ansari, 2009), female maquiladora (i.e., assembly plant) workers located along the Mexico-U.S. border (Liu et al., 1999), Taiwanese workers (Wang et al., 1998), and U.S. truck drivers (Guan et al., 2012). Some of these surveys have involved threedimensional body scans of populations such as adult wheelchair users (Paquet \& Feathers, 2004). A few studies have examined the impact on anthropometric variability of human laterality (i.e., left- or right- handedness) and gender (Mohammed, 2005), age (Annis, 1996; Jarosz, 1999; Pennathur \& Dowling, 2003; Hu et al., 2007), and race/ethnicity (Imrhan et al., 1993).

Summary statistics and the values of anthropometry at a few key percentiles are not directly usable in detailed accommodation analyses and design decision-making. There are four main reasons for this. First, as discussed in the previous paragraphs, this information describes a population's body dimensions at only a few percentiles; well-informed decision-making requires the availability of "higher-resolution" information for the entire population. Second, actual distributions of a population's body measures are likely to be non-Gaussian (Vasu \& Mital, 2000), which disallows the use of Gaussian tools (e.g., z-scores) in conjunction with this information to calculate the values at different percentiles. Third, summary statistics describe the state of a population's anthropometry at a certain point in time; secular trends could significantly alter these characteristics over a period of time (Drury, 2008). Fourth, separate sets of information for each body dimension fail to capture the correlations between different body measures; the direct use of this information in multivariate analyses can result in lower-than-desired accommodation of the user population (Moroney \& Smith, 1972).

\subsection{Anthropometry synthesis}

The aforementioned problems associated with the dearth of comprehensive anthropometric data for populations may be overcome through the use of anthropometry synthesis techniques. A number of techniques have been put forth over the years. These include methods based on proportionality constants, linear regression, regression with residual variance, and principal components analysis. The foundations of these methods are the correlations between body dimensions. This knowledge can be used along with data about a few basic body measures (e.g., stature and BMI - body mass index, a normalized ratio of weight for stature) to generate databases of the anthropometry that is relevant to the design effort. Each of these methods 

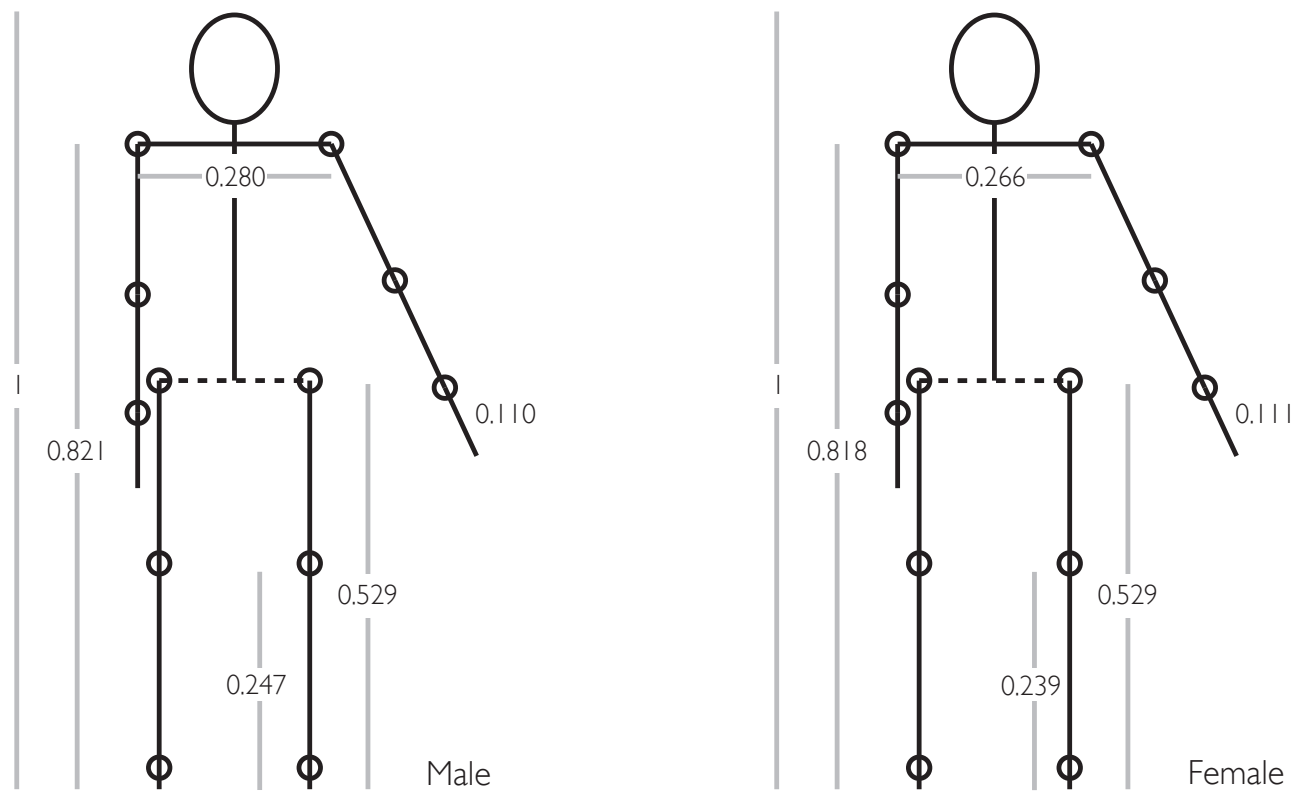

Figure 1: Some proportionality constants calculated for the female and male ANSUR populations.

assumes that relationships between measures in one population (e.g., the ratio of sitting height to stature) will exist in another.

Anthropometry synthesis methods adopt different approaches to utilize these correlations in synthesizing body measure data. Proportionality constantsbased techniques such as the ones proposed by Drillis \& Contini (1966) involve ratios of all body dimensions to stature; the anthropometry generated for every individual in the user population adheres to the fixed set of proportionality constants. Figure 1 shows some proportionality constants calculated for the ANSUR population. Despite their inherent simplicity, proportionality constants-based approaches are limited by certain invalid assumptions, including: a) all individuals are similarly proportioned, b) all body measures of an individual are of the same percentile, and c) independently accommodating for each body measure yields the desired accommodation level for the user population.

Body proportions are known to vary across individuals in a population (Ruff, 2002). Furthermore, the body dimensions that comprise an individual in a population are likely to represent different percentile values in their re- 
spective anthropometric distributions (Roebuck, 1995). The regression-based method presented by Nadadur \& Parkinson (2009) is capable of overcoming these limitations through the reincorporation into the synthesis model of the component of anthropometric variability that is unrelated to the predictor variable. This is represented by the residual variance (i.e., the squared value of standard error of variance) of the regression model relating the required body measure to the predictors, which are usually stature and BMI.

Regression-based methods require comprehensive data about the predictor variables - usually stature and BMI - for the target population. However, only descriptive statistics about anthropometry are available for a vast majority of possible target populations. A means to overcoming the unavailability of detailed predictor data involves first using descriptive statistical information to estimate the underlying stature and BMI distributions, then sampling from these distributions to create stature and BMI datasets for the population. This was the approach adopted by de Vries et al. (2011) to generate the requisite stature and BMI dataset of a U.S. truck driver population. This procedure is one of the bases for the anthropometry synthesis approach that is proposed in this paper.

\subsection{Anthropometric databases in virtual fitting}

References have been made in the preceding subsections about the value of accurately synthesized anthropometric databases for improved design decisionmaking capability. The widely applied practice of virtual fitting is an illustrative example to this point.

Virtual fitting allows for the simulation of user-product interactions (Chapman, 2011). The process involves formulating design constraints between various product specifications and their corresponding relevant body dimensions and postures (Godwin et al., 2007). When utilized in conjunction with detailed anthropometric data for the population of interest, these constraints help to evaluate the impact of different design specifications on the accommodation level afforded by the product. Efficient virtual fitting techniques make for the selection of product specifications that will achieve the desired accommodation level of the target population at minimal resource cost (Garneau \& Parkinson, 2009).

Virtual fitting may also be combined with the techniques of population modeling and posture prediction (Case et al., 2001; Kee \& Karwowski, 2001; Garneau \& Parkinson, 2010), which help to simulate user behavior. Together, these methods allow for predictions of the user's preferences (e.g., posture, 
reach) when interacting with the product; models of user behavior may be developed based on experiments that study user-product interacts (e.g., Kolich \& Taboun (2004); Reed (2005); O'Sullivan \& Gallwey (2005)). The tremendous capabilities of virtual fitting-based approaches are contingent on the availability of accurate anthropometric databases for the population of interest.

\subsection{Research focus}

This paper presents an alternative to the regression-based approaches to estimating anthropometry. The proposed percentile combinations datasets (PCD) method draws on descriptive statistics (summary statistics and bypercentile values) in synthesizing a detailed database of anthropometry for a virtual population of individuals. The synthesis is in effect a "reverseengineering" process to recreate the underlying data for which the descriptive statistics were computed. The statistical equivalence achieved between the virtual and actual user populations allows for the utilization of the synthesized database in in-depth accommodation analyses for design decisionmaking; this is not possible with only descriptive statistics. The PCD method thereby allows for the effective use of the many existing research reports and articles that contain descriptive statistical information (summary statistics and by-percentile values) for a wide variety of global populations. The raw anthropometric data behind these descriptive statistics, which are not made publicly available for a variety of reasons, may thus be "reverse-engineered" and utilized in design efforts.

\section{Methodology}

The PCD technique draws on information about body measures at different percentiles, and yields a synthesized anthropometric database for the population. The method consists of a preliminary step involving the analysis

of a comprehensive and representative "reference" anthropometric database (e.g., ANSUR). This is followed by the three steps of collecting descriptive statistics, fitting anthropometric models, and sampling the distributions to yield the synthesized anthropometric database for the target population. These steps are introduced and presented in this section.

\subsection{Preliminary Step: Reference database analysis}

As discussed in the previous section, the body proportions of individuals vary within and across populations. For a sufficiently large population, 


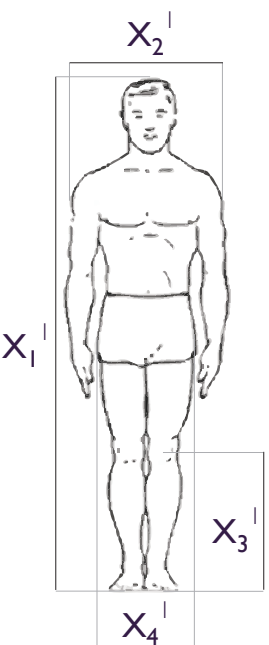

Individual |

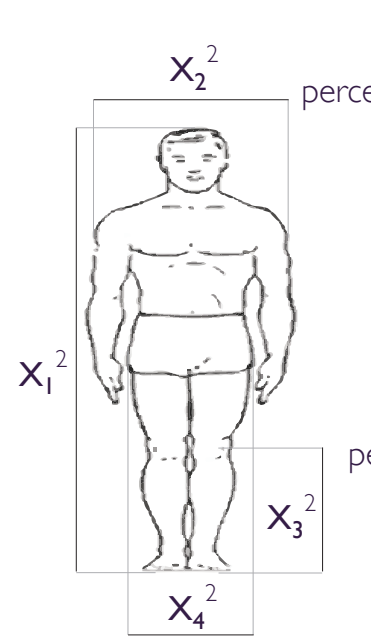

Individual 2

$\left\{\mathrm{X}_{1}{ }^{2}, \mathrm{X}_{2}{ }^{2}, \mathrm{X}_{3}{ }^{2}, \mathrm{X}_{4}{ }^{2}\right\}$

Anthropometric distributions

$\left\{\mathrm{X}_{1}{ }^{\prime}, \mathrm{X}_{2}{ }^{\prime}, \mathrm{X}_{3}{ }^{\prime}, \mathrm{X}_{4}{ }^{\prime}\right\}$
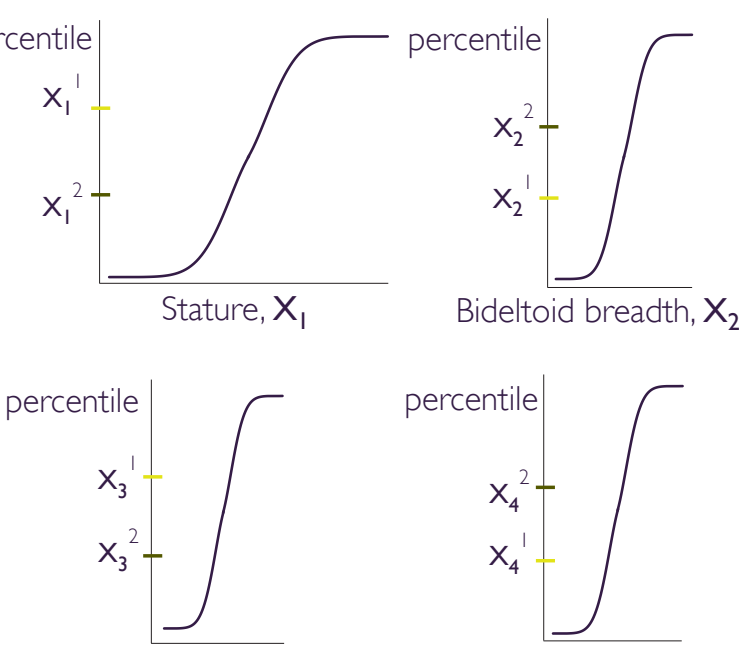

Knee height, $\mathrm{X}_{3}$

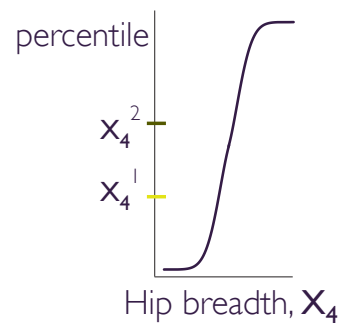

Figure 2: An illustration of the different anthropometric "percentile sets" of body measures 1 (stature), 2 (bideltoid breadth), 3 (knee height), and 4 (hip breadth) that constitute individuals 1 and 2 .

body measures that are significantly correlated to stature and weight vary according to distributions that are approximately normal (Gaussian) or lognormal in nature, respectively (Brainard \& Burmaster, 1992). The value of a body measure for an individual is then a particular percentile in its distribution. Accordingly, an individual can be thought of as consisting of combinations of percentiles of anthropometry. For instance, as illustrated in Figure 2, an Individual 1 may be constituted of the $\mathrm{X}_{1}{ }^{1}, \mathrm{X}_{2}{ }^{1}, \mathrm{X}_{3}{ }^{1}$, and $\mathrm{X}_{4}{ }^{1}$ percentiles of body measures $1,2,3$, and 4 (stature, bideltoid breadth, knee height, and hip breadth, respectively) as sampled from their respective distributions. Similarly, Individual 2 may be comprised of the $\mathrm{X}_{1}{ }^{2}, \mathrm{X}_{2}{ }^{2}, \mathrm{X}_{3}{ }^{2}$, and $\mathrm{X}_{4}{ }^{2}$ percentiles of the four body measures. Therefore, Individuals 1 and 2 can be represented as anthropometric "percentile sets" of the form $\left\{\mathrm{X}_{1}{ }^{1}\right.$, $\left.\mathrm{X}_{2}{ }^{1}, \mathrm{X}_{3}{ }^{1}, \mathrm{X}_{4}{ }^{1}\right\}$ and $\left\{\mathrm{X}_{1}{ }^{2}, \mathrm{X}_{2}{ }^{2}, \mathrm{X}_{3}{ }^{2}, \mathrm{X}_{4}{ }^{2}\right\}$, respectively. In general, for the $\mathrm{m}^{\text {th }}$ individual in a population, the percentile set of $\mathrm{n}$ body measures can be in the form of: $\left\{\mathrm{X}_{1}{ }^{\mathrm{m}}, \mathrm{X}_{2}{ }^{\mathrm{m}}, \mathrm{X}_{3}{ }^{\mathrm{m}}, \mathrm{X}_{4}{ }^{\mathrm{m}}, \ldots, \mathrm{X}_{n}{ }^{\mathrm{m}}\right\}$.

Not only can the percentile sets capture the inter-relationships of the different body measures, but they can also ensure that the proportions of body 
dimensions estimated for individuals are realistic. This reduces, for instance, the possibility of thigh circumference being greater than hip circumference for an individual. Additionally, the percentile sets can be used in conjunction with information about the anthropometric distributions in order to calculate the values of the required body measures for individuals in a population. This is the underlying process implemented in the PCD method.

The method is based on a fundamental assumption: that the complete dataset of percentile combinations (Equation 1) that is calculated for all the individuals in a population is similar to the datasets calculated for other populations. In other words, in every sufficiently large population, there exist people similar to Individuals 1 and 2 (Figure 2) whose 4 body dimensions can be represented by the percentile sets $\left\{\mathrm{X}_{1}{ }^{1}, \mathrm{X}_{2}{ }^{1}, \mathrm{X}_{3}{ }^{1}, \mathrm{X}_{4}{ }^{1}\right\}$ and $\left\{\mathrm{X}_{1}{ }^{2}, \mathrm{X}_{2}{ }^{2}, \mathrm{X}_{3}{ }^{2}\right.$, $\left.\mathrm{X}_{4}^{2}\right\}$, respectively. For example, suppose the stature $\left(\mathrm{X}_{1}\right)$, bideltoid breadth $\left(\mathrm{X}_{2}\right)$, knee height $\left(\mathrm{X}_{3}\right)$, and hip breadth $\left(\mathrm{X}_{4}\right)$ for an individual $\mathrm{m}$ in ANSUR are of the $87.3^{\text {th }}, 56.9^{\text {th }}, 76.2^{\text {th }}$, and $64.8^{\text {th }}$ percentiles, respectively, that individual can be represented as: $\left\{\mathrm{X}_{1}{ }^{\mathrm{m}}, \mathrm{X}_{2}{ }^{\mathrm{m}}, \mathrm{X}_{3}{ }^{\mathrm{m}}, \mathrm{X}_{4}{ }^{\mathrm{m}}\right\}=\left\{87.3^{\text {th }}, 56.9^{\text {th }}\right.$, $\left.76.2^{\text {th }}, 64.8^{\text {th }}\right\}$. Furthermore, based on the assumption made in this method, there exists an individual $\mathrm{m}$ in some other population (e.g., India, China, Kenya) whose stature, bideltoid breadth, knee height, and hip breadth can respectively be represented as: $\left\{\mathrm{X}_{1}{ }^{{ }^{\prime}}, \mathrm{X}_{2}{ }^{{ }^{\prime}}, \mathrm{X}_{3}{ }^{\mathrm{m}^{\prime}}, \mathrm{X}_{4}{ }^{\mathrm{m}^{\prime}}\right\}=\left\{87.3^{\text {th }}, 56.9^{\text {th }}\right.$, $\left.76.2^{\text {th }}, 64.8^{\text {th }}\right\}$.

$$
\begin{aligned}
& X_{1}{ }^{1}, X_{2}{ }^{1}, X_{3}{ }^{1}, X_{4}{ }^{1}, \cdots, X_{n}{ }^{1} \\
& X_{1}{ }^{2}, X_{2}{ }^{2}, X_{3}{ }^{2}, X_{4}{ }^{2}, \cdots, X_{n}{ }^{2} \\
& \quad \cdot \\
& \quad \cdot \\
& \quad \cdot \\
& X_{1}{ }^{\mathrm{m}}, X_{2}{ }^{\mathrm{m}}, X_{3}{ }^{\mathrm{m}}, X_{4}{ }^{\mathrm{m}}, \cdots, X_{n}{ }^{\mathrm{m}}
\end{aligned}
$$

Note that the assumption does not imply that individuals in every population are similarly sized or similarly proportioned. The assumption does not even imply similar correlation coefficients for the anthropometry of two different populations. Instead, it means that there are certain patterns of multidimensional correlations in anthropometry that are similar across populations; the percentile combination datasets capture these patterns. Therefore, given a population's unique mix of anthropometric distributions, the 
PCDs can be applied to synthesize unique anthropometric data for that population. Since the characteristics of the underlying body measures and their distributions vary across populations, sampling from those distributions using the same percentile sets will result in the generation of individuals who are differently proportioned across populations.

A simple test of the aforementioned assumption was conducted in the context of the U.S. military and Japanese youth, represented by ANSUR (Gordon et al., 1989) and AIST (National Institute of Bioscience and HumanTechnology, Nippon Shuppan Service, Tokyo, 1996), which are introduced in greater detail in Sections 3.1 and 3.3, respectively. These are significantly different populations in terms of anthropometric variability (Section 4). The divergence of the two populations notwithstanding, the fundamental assumption of the PCD approach is that percentile combination datasets are capable of capturing the patterns of variation of the underlying inter-anthropometric correlations that tend to be consistent across populations. Tables 1 and 2 were utilized to test this assumption in the context of the chosen reference and target populations: ANSUR and Japan, respectively.

Correlation matrices were separately generated for the percentile values of anthropometry calculated for the female and male Japanese populations. A total of 28 anthropometry pairs were identified in each correlation matrix as having high correlation coefficients $(\geqslant 0.75)$. Using similar percentiles-based correlation matrices for the female and male ANSUR populations, percentage differences were calculated between the Japanese and ANSUR populations for the 28 identified anthropometry pairs. As seen in Tables 1 and 2, the majority of the percentage differences are low $(\leqslant 5 \%): 21$ and 22 for the female and male data, respectively. There are a total of only 4 high percentage differences $(\geqslant 10 \%)$ : 1 in the female dataset and 3 in the male dataset. These observations are indicative of a general consistency in underlying percentilebased correlations for the significantly correlated anthropometry pairs across the two populations. This is an indication that the underlying assumption of the PCD approach may be correct in the context of the Japanese and ANSUR populations.

Given the assumption of the proposed approach, the reference database in the PCD method (Figure 3) should be selected for its representativeness (i.e., capturing the demographic and anthropometric variations of the underlying population) and comprehensiveness (i.e., containing all the required body dimensions). ANSUR satisfies these properties, and is also well-known and widely used; for this reason it is used as the source database for the percentile 
Table 1: Percentage differences between correlation coefficients of the percentile combination datasets for ANSUR (ANS) and actual (Act) and synthesized (Syn) female Japanese body dimensions. Values are presented only for the 28 anthropometry pairs which exhibit high correlations $(\geqslant 0.75)$ in the actual Japanese population. Values are percentages.

\begin{tabular}{|c|c|c|c|c|c|c|c|c|c|}
\hline & $\begin{array}{l}\text { Butt- } \\
\text { knee } \\
\text { lnth }\end{array}$ & $\begin{array}{l}\text { Butt- } \\
\text { pop } \\
\text { lnth }\end{array}$ & $\begin{array}{c}\text { Knee } \\
\text { ht, } \\
\text { sit }\end{array}$ & $\begin{array}{l}\text { Pop } \\
\text { ht, } \\
\text { sit }\end{array}$ & $\begin{array}{l}\text { Sit } \\
\text { ht }\end{array}$ & Stat & $\begin{array}{c}\text { Troc } \\
\text { ht }\end{array}$ & Wght & BMI \\
\hline \multicolumn{10}{|c|}{ Acromial ht } \\
\hline $\begin{array}{l}\text { Act-ANS } \\
\text { Act-Syn }\end{array}$ & 1.7 & 2.5 & 2.9 & 2.2 & 9.6 & 1.3 & 0.6 & - & - \\
\hline \multicolumn{10}{|c|}{ Bideltoid brth } \\
\hline Act-ANS & - & - & - & - & - & - & - & 4.7 & 10.4 \\
\hline Act-Syn & - & - & - & - & - & - & - & 4.7 & 10.5 \\
\hline \multicolumn{10}{|c|}{ Butt-knee lnth } \\
\hline Act-ANS & - & 0.2 & 4.4 & 11.9 & - & 5.0 & 0.1 & - & - \\
\hline Act-Syn & - & 0.2 & 4.5 & 11.9 & - & 5.0 & 0.1 & - & - \\
\hline \multicolumn{10}{|c|}{ Butt-popliteal lnth } \\
\hline Act-ANS & - & - & 7.0 & 4.4 & - & 6.7 & 0.6 & - & - \\
\hline Act-Syn & - & - & 7.0 & 4.4 & - & 6.6 & 0.6 & - & - \\
\hline \multicolumn{10}{|c|}{ Hip brth, sit } \\
\hline Act-ANS & - & - & - & - & - & - & - & 5.0 & 3.6 \\
\hline Act-Syn & - & - & - & - & - & - & 0 & 5.0 & 3.6 \\
\hline \multicolumn{10}{|c|}{ Knee ht, sit } \\
\hline Act-ANS & - & - & - & 0.7 & - & 1.8 & 9.4 & - & - \\
\hline Act-Syn & - & - & - & 0.7 & - & 1.8 & 9.4 & - & - \\
\hline \multicolumn{10}{|c|}{ Popliteal ht, sit } \\
\hline Act-ANS & - & - & - & - & - & 4.9 & 3.9 & - & - \\
\hline Act-Syn & - & - & - & - & - & 4.9 & 3.9 & - & - \\
\hline \multicolumn{10}{|l|}{ Sitting ht } \\
\hline Act-ANS & - & - & - & - & - & 7.0 & - & - & - \\
\hline Act-Syn & - & - & - & - & - & 7.0 & - & - & - \\
\hline \multicolumn{10}{|l|}{ Stature } \\
\hline Act-ANS & - & - & - & - & - & - & 4.2 & - & - \\
\hline Act-Syn & - & - & - & - & - & - & 4.2 & - & - \\
\hline \multicolumn{10}{|l|}{ Weight } \\
\hline Act-ANS & - & - & - & - & - & - & - & - & 0.1 \\
\hline Act-Syn & - & - & - & - & - & - & - & - & 0.1 \\
\hline
\end{tabular}


Table 2: Percentage differences between correlation coefficients of the percentile combination datasets for ANSUR (ANS) and actual (Act) and synthesized (Syn) male Japanese body dimensions. Values are presented only for the 28 anthropometry pairs which exhibit high correlations $(\geqslant 0.75)$ in the actual Japanese population. Values are percentages.

\begin{tabular}{|c|c|c|c|c|c|c|c|c|c|}
\hline & $\begin{array}{l}\text { Butt- } \\
\text { knee } \\
\text { lnth }\end{array}$ & $\begin{array}{c}\text { Butt- } \\
\text { pop } \\
\text { lnth }\end{array}$ & $\begin{array}{l}\text { Knee } \\
\text { ht, } \\
\text { sit }\end{array}$ & $\begin{array}{l}\text { Pop } \\
\text { ht, } \\
\text { sit }\end{array}$ & $\begin{array}{l}\text { Sit } \\
\text { ht }\end{array}$ & Stat & $\begin{array}{c}\text { Troc } \\
\text { ht }\end{array}$ & Wght & BMI \\
\hline \multicolumn{10}{|c|}{ Acromial ht } \\
\hline Act-ANS & 2.5 & 0.6 & 4.9 & 4.3 & 16.3 & 0.2 & 0.1 & - & - \\
\hline Act-Syn & 2.5 & 0.6 & 4.9 & 4.3 & 16.3 & 0.2 & 0.1 & - & - \\
\hline \multicolumn{10}{|c|}{ Bideltoid brth } \\
\hline Act-ANS & - & - & - & - & - & - & - & 1.3 & 5.7 \\
\hline Act-Syn & - & - & - & - & - & - & - & 1.3 & 5.7 \\
\hline \multicolumn{10}{|c|}{ Butt-knee lnth } \\
\hline Act-ANS & - & 1.7 & 0.3 & 0.1 & - & 1.7 & 2.2 & - & - \\
\hline Act-Syn & - & 1.7 & 0.3 & 0.1 & - & 1.7 & 2.2 & - & - \\
\hline \multicolumn{10}{|c|}{ Butt-popliteal lnth } \\
\hline Act-ANS & - & - & 0.8 & 2.4 & - & 1.2 & 1.5 & - & - \\
\hline Act-Syn & - & - & 0.8 & 2.4 & - & 1.2 & 1.5 & - & - \\
\hline \multicolumn{10}{|c|}{ Hip brth, sit } \\
\hline Act-ANS & - & - & - & - & - & - & - & 5.8 & 17.2 \\
\hline Act-Syn & - & - & - & - & - & - & - & 5.8 & 17.2 \\
\hline \multicolumn{10}{|c|}{ Knee ht, sit } \\
\hline Act-ANS & - & - & - & 5.8 & - & 3.2 & 2.2 & - & - \\
\hline Act-Syn & - & - & - & 5.8 & - & 3.2 & 2.2 & - & - \\
\hline \multicolumn{10}{|c|}{ Popliteal ht, sit } \\
\hline Act-ANS & - & - & - & - & - & 1.8 & 3.6 & - & - \\
\hline Act-Syn & - & - & - & - & - & 1.8 & 3.6 & - & - \\
\hline \multicolumn{10}{|l|}{ Sitting ht } \\
\hline Act-ANS & - & - & - & - & - & 12.7 & - & - & - \\
\hline Act-Syn & - & - & - & - & - & 12.7 & - & - & - \\
\hline \multicolumn{10}{|l|}{ Stature } \\
\hline Act-ANS & - & - & - & - & - & - & 0.1 & - & - \\
\hline Act-Syn & - & - & - & - & - & - & 0.1 & - & - \\
\hline \multicolumn{10}{|l|}{ Weight } \\
\hline Act-ANS & - & - & - & - & - & - & - & - & 3.9 \\
\hline Act-Syn & - & - & - & - & - & - & - & - & 3.9 \\
\hline
\end{tabular}




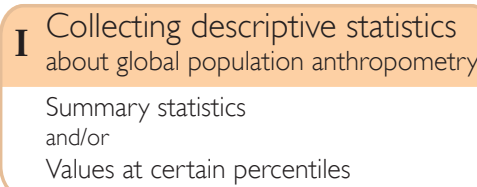

I Collecting descriptive statistics
about global population anthropometry
Summary statistics
and/or
Values at certain percentiles
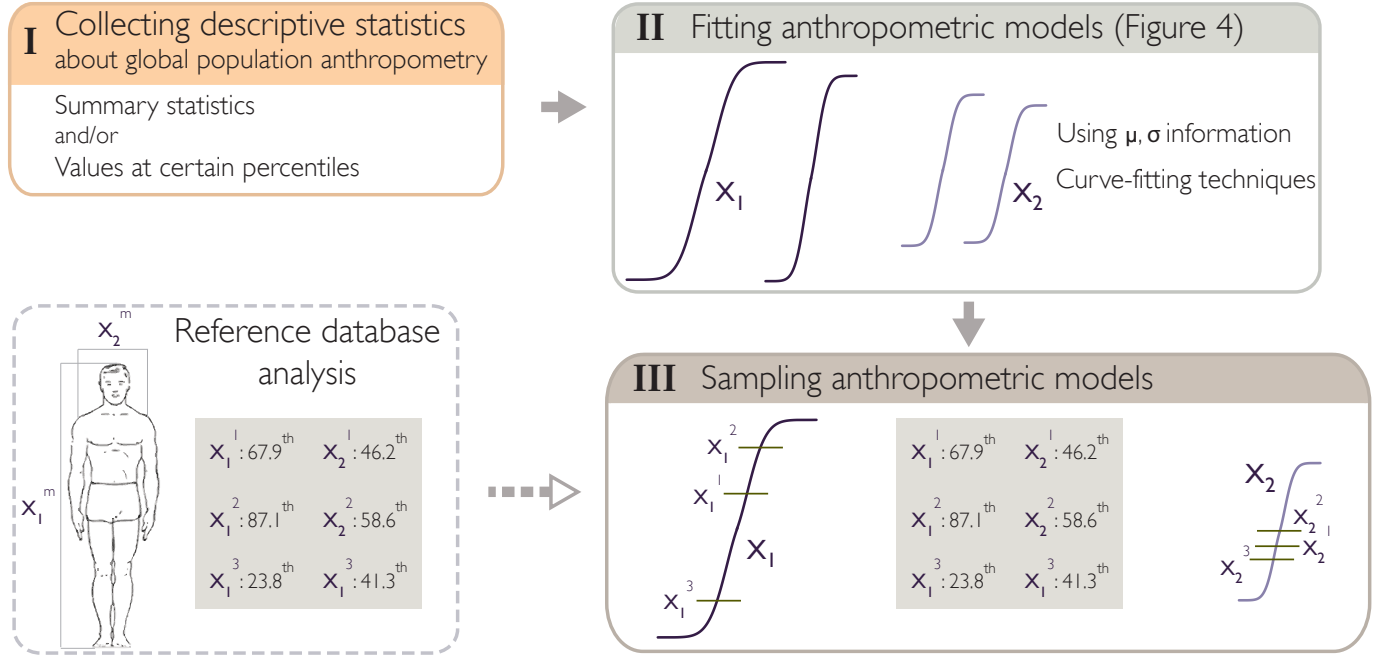

Figure 3: The proposed method for synthesizing anthropometry for target user populations.

combination dataset in this example. The percentile value of every body measure for each ANSUR individual is calculated. This analysis results in a dataset consisting of the percentile set of every individual. Separate datasets are created for the female and male ANSUR participants.

\subsection{Step I: Collecting descriptive statistics}

The first step of the method involves locating and gathering information about the population of interest. This information is typically in one or both of two forms: a) summary statistics, which can include the means and standard deviations of the anthropometric distributions for that population and b) values of the anthropometry at certain key percentiles (e.g., $1^{\text {st }}, 5^{\text {th }}, 50^{\text {th }}$, $\left.95^{\text {th }}, 99^{\text {th }}\right)$. The first form of information would require the assumption of a particular distribution (e.g., Gaussian or lognormal) for the entire range of anthropometry; the accuracy of this assumption varies with population composition and the specific measure being modeled. The second form can better allow for the modeling of non-normal variation of anthropometry across the different percentiles. This makes for improved estimation of the underlying distribution (Section 2.3).

As mentioned in Section 1, there are numerous sources of such anthropometric information. These can be divided into two broad categories: a) reports that are the result of large-scale surveys or data acquisition initiatives 
by organizations and agencies and b) publications on smaller-scale surveys by various research groups. The ISO/TR 7250-2 standards (ISO, 2010) by the International Organization for Standardization is an example of the former category of sources, and consists of information about the working age populations of eight nations. The State Bureau of Technical Supervision (1989) is another example of this type of report, and is the result of a survey of around 20,000 individuals by the Chinese State Bureau of Technical Supervision. Gite et al. (2009) provides the means and standard deviations of the body dimensions of agricultural worker populations in each state in India. Barroso et al. (2005) presents summary statistical information that is calculated based on a survey of 399 female and 492 male Portuguese workers.

Smaller-scale surveys tend to be more focused on certain segments of populations. These include Mexican female assembly plant workers (Liu et al., 1999), Bahraini school children between 6 and 12 years of age (Mokdad \& Al-Ansari, 2009), and Algerian date farmers (Mokdad, 2002).

\subsection{Step II: Fitting anthropometric models}

The second step of the proposed method entails fitting models to the anthropometric information obtained in the previous step. The models are required in Step III of the method for the calculation of anthropometric values across a range of percentiles that is not limited to just the ones provided in the sources collected in Step I of the method.

The simplest solution to the model-fitting problem is to assume that every body measure adheres to a Gaussian distribution. In general, in the sources obtained in Step I, the means of the distributions are assumed equivalent to the $50^{\text {th }}$ percentile, and the standard deviations are subsequently calculated using the z-score formula for normal distributions:

$$
\mathbf{x}=\mu+\mathbf{z} \cdot \sigma
$$

where $\mathbf{x}$ : value of the body measure at a certain percentile, $\mathbf{z}$ : the $\mathrm{z}$-score of the normal distribution at that percentile, $\mu$ : the mean of the anthropometric distribution, assumed equivalent to the $50^{\text {th }}$ percentile, and $\sigma$ : the standard deviation of the distribution. Values of $\mu$ and $\sigma$ can then be used along with appropriate z-scores to estimate the required percentile values of that body measure.

However, this solution can result in significant errors in the synthesized anthropometry. In reality, the underlying anthropometric data are unlikely 
to be perfectly Gaussian. A procedure based on using z-scores is therefore unlikely to be accurate across the entire range of percentiles of the anthropometry. Additionally, the assumption of the mean being equal to the $50^{\text {th }}$ percentile is itself invalid for non-Gaussian distributions.

In order to avoid the aforementioned limitations, a number of techniques can be utilized to fit optimal models to the available anthropometric information. A technique that is simple, efficient, and easy to implement is outlined and demonstrated here. It involves splitting the underlying distribution of every body dimension into two halves - above and below the $50^{\text {th }}$ percentile - and independently fitting a different Gaussian curve to each half. The application of this procedure is described in greater detail in Section 3.3.

\subsection{Step III: Sampling anthropometric models}

Step II yields the distributions for the anthropometry of interest for the target population. The procedure for synthesizing anthropometry for each individual consists of two basic stages.

First, locate the set of percentile combinations (e.g., $\left\{\mathrm{X}_{1}{ }^{\mathrm{m}}, \mathrm{X}_{2}{ }^{\mathrm{m}}, \mathrm{X}_{3}{ }^{\mathrm{m}}\right.$, $\left.\mathrm{X}_{4}{ }^{\mathrm{m}}, \ldots, \mathrm{X}_{n}{ }^{\mathrm{m}}\right\}$ ) of the $\mathrm{n}$ body measures for the individual $m$ (calculated in Step I). Second, for each percentile value (e.g., $\mathrm{X}_{1}{ }^{\mathrm{m}}$ ), sample the corresponding distribution (e.g., the distribution of anthropometry $\mathrm{X}_{1}$ ) to obtain

the actual value of the body measure (Figure 2). These sampled values of body dimensions together constitute the anthropometry for the individual. This procedure can be repeated for each individual in the population, and results in the required synthesized anthropometric database for that population.

In the following section, the proposed methodology is demonstrated and tested with two populations of interest.

\section{Case study}

The proposed method is demonstrated in the following case study, which involved first synthesizing anthropometric data for a known population, then comparing the synthesized and actual anthropometry. The case study makes use of two comprehensive databases, one as the reference population and the other as the target population for which anthropometry must be synthesized. The descriptive statistics required for Step I of the method were calculated based on the actual data available for the target population. The descriptive statistics were utilized as inputs to the PCD method. The anthropometric 
data thereby synthesized were compared with the actual data of the target population to check the accuracy of the method.

The body measures selected for this study were 13 in number, and included 9 measures of length and 4 measures of breadth or weight. The body dimensions were: acromial height, bideltoid breadth, buttock-knee length, buttock-popliteal length, hand length, seated hip breadth, seated knee height, seated popliteal height, sitting height, stature, trochanteric height, weight, and BMI.

\subsection{Preliminary step: ANSUR analysis}

ANSUR provides 132 body dimensions for 2208 females and 1774 males. The female and male populations were considered separately for this analysis, which consisted of the process illustrated in Figure 2 and described in Section 2.1.

The percentile rank of each of the 13 chosen body measures (i.e., the percentile set) was calculated for every individual in the female and male

populations. These female and male percentile sets were compiled to yield a gender-based percentile dataset for the population.

\subsection{Step I: Collecting information}

The target population for this case study was civilian Japanese youth (National Institute of Bioscience and Human-Technology, Nippon Shuppan Service, Tokyo, 1996). This anthropometric database was compiled in the 1991-1992 period, and contains data for 255 different body dimensions. The survey consists of two discrete age groups of individuals: 18-29 years and greater than 60 years. The former category is composed of 400 individuals in total, 200 female and 200 male; these individuals are referred to as youth populations in this study. The latter category is comprised of 50 females and 50 males, and was not included in the database utilized in this demonstration.

The 400 Japanese youth were included in this case study, and the anthropometry synthesis methodology was applied separately for the female and male groups. For the remainder of this article, the term "Japanese population" is used to refer to either of these within-gender groups; they are explicitly referred to as female or male when required.

The anthropometry and its variability was expected to be significantly different for the ANSUR and Japanese populations, since the body proportions (e.g., sitting height to stature) and sizes are known to be different across these populations (Tilley \& Dreyfuss, 2001). The effectiveness of the 

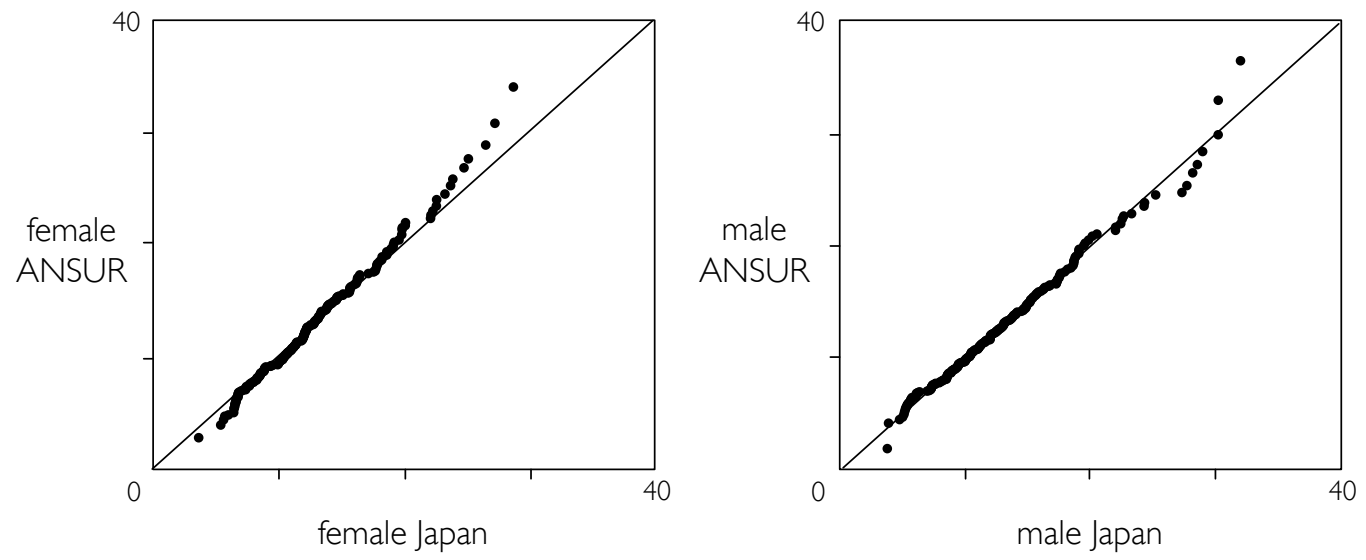

Figure 4: Q-Q plots of the Mahalanobis distances calculated for the female and male ANSUR and Japanese youth populations using their respective percentile combinations datasets. The distances for each population are calculated about its own Mahalanobis centroid.

proposed methodology for both these populations would be indicative of its robustness and versatility.

A useful metric for comparing the underlying variation within datasets is the Mahalanobis distance (Mahalanobis, 1936). This metric can be used to statistically describe multidimensional data, with the advantage over Euclidean distances being that Mahalanobis distances take into account differing variances in each dimension while also considering possible inter-dimensional correlations. Accordingly, Mahalanobis distances are applied to supplement the observations from Tables 3-6.

Figure 4 is a Q-Q (i.e., quantile-quantile) plot of the Mahalanobis distances calculated for the ANSUR and Japanese youth populations using their respective percentile combinations datasets. The distances were calculated independently for each population with respect to its own multidimensional centroid. Q-Q plots are comparisons of pairs of probability distributions. They consist of points representing the values of the two distributions across the different quantiles. Figure 4 consists of separate plots that compare the distances for the female and male populations. If the two distributions being compared are similar, the points in their Q-Q plot will lie approximately on a 45 degree line. The points in the plots for the female and male populations appear to correspond closely to the line.

A relatively lower degree of convergence between the points and the 45 de- 
gree line was observed at the highest quantiles. This divergence could imply that for the Japan and ANSUR populations, anthropometric variation as quantified by the Mahalanobis distance is divergent at the tails of the distributions. This degree of divergence was not expected to impact the overall effectiveness of the PCD method in the context of the two populations.

The Wilcoxon test was used to quantitatively complement the Q-Q plotsbased visual observations of the underlying variances in the populations' anthropometry; the Mahalanobis distances utilized in the plots were compared through this statistical procedure. The $\mathrm{W}$-values for the female and male Japan-ANSUR comparisons were found to be 211665 ( $\mathrm{p}$-value=0.7863) and 189361 ( $\mathrm{p}$-value $=0.6938)$, respectively. These results imply that the differences in the 13 chosen body dimensions for the two populations may not be significant.

The Mahalanobis distance comparisons indicate that there may be underlying statistical patterns that are valid across the ANSUR and Japanese youth populations. These patterns indicate the similarity of PCDs for the ANSUR and Japan populations; this is the basis for the application of the PCD method in this context.

As discussed in Section 2.2, the input for the method consists of the values of the required body measures at key percentiles. The key percentiles considered in this study were the $1^{\text {st }}, 5^{\text {th }}, 50^{\text {th }}, 95^{\text {th }}$, and $99^{\text {th }}$, which are the same as the standard set of percentiles included in the ISO/TR 7250-2 report (ISO, 2010). Accordingly, the values of the 13 selected body measures were calculated at these 5 key percentiles for the Japanese populations. These percentile values were the inputs for the anthropometric distribution generation phase (Step II) of the methodology.

\subsection{Step II: Fitting anthropometric models}

The proposed procedure for fitting the required anthropometric models was mentioned in Section 2.3 and is illustrated in Figure 5. While the underlying anthropometric distribution may be approximately Gaussian in nature, it is likely to differ in the lower and upper halves. For this reason, the procedure involved generating separate Gaussian models based on the lower and upper halves of percentile values, which are $\leqslant 50^{\text {th }}$ percentile (i.e., $1^{\text {st }}, 5^{\text {th }}$, $50^{\text {th }}$ ) and $\geqslant 50^{\text {th }}$ percentile (i.e., $50^{\text {th }}, 95^{\text {th }}, 99^{\text {th }}$ ).

For a given body measure, the procedure consisted of two main stages. Stage $\mathbf{i}$ was commenced with the calculation of the initial mean $\left(\mu_{I}\right)$ of the body measure as being equal to the $50^{\text {th }}$ percentile value. This initial mean 
i Generating multiple distributions using means and standard deviations that vary within specified boundaries

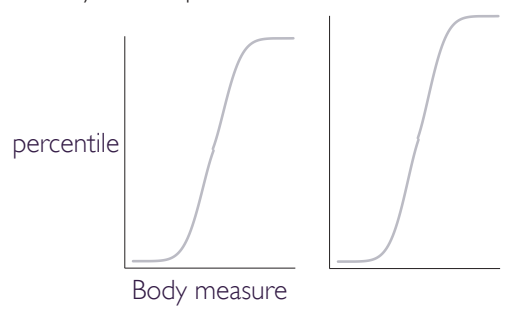

ii Calculating the error in fit

at the key percentiles (Equation 3)

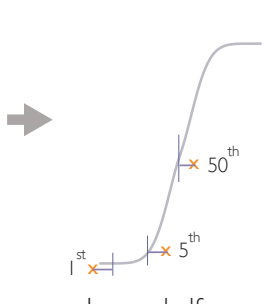

Lower half

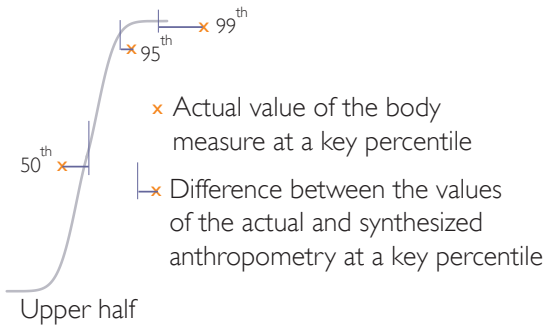

Figure 5: The procedure to fitting Gaussian anthropometric models (Step II of the proposed anthropometry synthesis methodology) for a population based on known values of the body measures at certain key percentiles (Equation 4).

value was used along with the $5^{\text {th }}$ and $95^{\text {th }}$ percentile z-scores $(\mathbf{z})$ to compute separate initial standard deviation values for the lower $\left(\sigma_{I, 5^{t h}}\right)$ and upper $\left(\sigma_{I, 95^{t h}}\right)$ halves of the body measure. The equation utilized for this purpose was derived from Equation 2:

$$
\sigma_{I}=\left(\mathbf{x}-\mu_{I}\right) / \mathbf{z}
$$

where $\mathbf{x}$ : the value of the body measure at the $5^{\text {th }}$ percentile for the lower half and $95^{\text {th }}$ percentile for the upper half and $\mathbf{z}$ : the $\mathbf{z}$-score for Gaussian distributions at the $5^{\text {th }}$ percentile or $95^{\text {th }}$ percentile (-1.645 or 1.645 , respectively).

The values of $\mu_{I}$ and $\sigma_{I}$ were not directly utilized in the anthropometric models. Doing so would entail the assumption that the model was perfectly Gaussian, with the mean being equal to the $50^{\text {th }}$ percentile and the standard deviation being invariant throughout the distribution. As discussed in Section 2.3, these are unlikely to be valid assumptions.

Accordingly, $\mu_{I}, \sigma_{I, 5^{t h}}$, and $\sigma_{I, 95^{t h}}$ were used as the starting points for the model-fitting procedure. Constraints were specified for the variation of the mean and standard deviation around their initial values. Within these limits, a number of $\left[\mu_{I}, \sigma_{I, 5^{t h}}, \sigma_{I, 95^{t h}}\right]$ combinations were formed, and two Gaussian distributions were generated for each combination (Figure 5), one for the lower half $\left(\mu_{I}, \sigma_{I, 5^{t h}}\right)$ and one for the upper half $\left(\mu_{I}, \sigma_{I, 95^{t h}}\right)$.

Stage ii involved assessing the fit of each distribution pair (i.e., the lower and upper half distributions) from Stage $\mathbf{i}$ to the known percentile values of the body measure. The objective of this process was to minimize the absolute 
sum of the errors $(\epsilon)$ between the fitted and actual distributions at the key percentiles (Equation 4):

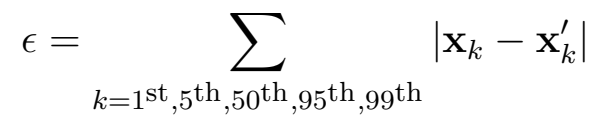

where $\mathbf{x}$ and $\mathbf{x}$ ' were respectively the actual and synthesized values of the body measure at percentile $k$. This process yielded different Gaussian models for the lower and upper halves of the body measure.

\subsection{Step III: Sampling anthropometric models}

The final step of the anthropometry synthesis method involved generating complete anthropometric databases for the Japanese populations using the female and male percentile datasets from the preliminary step (Section 3.1) and the models from Step II (Section 3.3).

As described in Section 2.1, every ANSUR individual was represented by a percentile set. The Japanese equivalent of that individual was synthesized by sampling the anthropometric models of the Japanese population for the required percentiles. For example, the individual $\mathrm{X}^{\mathrm{m}}$ in Figure 2 was synthesized for the Japanese population by sampling the Gaussian models of the $\mathrm{X}_{1}, \mathrm{X}_{2}, \mathrm{X}_{3}$, and $\mathrm{X}_{4}$ body measures of the Japanese population for the $\mathrm{X}_{1}{ }^{\mathrm{m}}$ th , $\mathrm{X}_{2}{ }^{\mathrm{m} \text { th }}, \mathrm{X}_{3}{ }^{\mathrm{m} \text { th }}$, and $\mathrm{X}_{4}{ }^{\mathrm{m}}$ th percentile values, respectively.

This procedure was repeated for every individual in the female and male Japanese populations, resulting in a complete anthropometric database for

either population. The accuracy of these data, and therefore the effectiveness of the proposed method, are examined in the following section.

\section{Results}

The anthropometry synthesis methodology proposed in Section 2 was demonstrated in Section 3 as being a simple procedure to implement. The required inputs for the methodology (Step I) consist of values of the target population's body measures at a set of key percentiles; this information is easily obtainable for numerous global user populations. The stages of model fitting (Step II) and model sampling (Step III) are basic in nature, and are therefore less likely to suffer from the problem of being well-suited to specific pairs of reference and target populations and less-suited for other population pairs. This makes them more likely to be suitable for application across a wide spectrum of populations. 
Tables 3, 4, 5, and 6 and Figure 4 are used to compare the ANSUR and Japanese populations to verify their divergence in terms of anthropometric variation and body proportions. Tables 3 and 4 contain the means, standard deviations, and the values of the 13 body measures at the key percentiles used in the synthesis method. These percentile values were directly calculated from the databases of actual and synthesized anthropometry, and not indirectly using the means, standard deviations, and appropriate z-scores in Equation 2. With the exception of hand length and sitting popliteal height, the two populations exhibit marked variation in their anthropometry, both in terms of their means and standard deviations and also across the range of considered percentiles.

The extent of variation of anthropometry are captured in Tables 3 and 4; however, this does not help to compare the underlying body proportions (e.g., sitting height to stature) of the populations, which represent a fundamental difference in the populations' anthropometry. A preliminary exploration of this is presented in Tables 5 and 6, which compare the body proportions of the female and male populations. The procedure involved in calculating the means and standard deviations contained in the tables consisted of two steps. First, the ratios of the different body dimensions to stature were computed for every individual in the population; this yielded the proportionality constants for each individual. Second, the dataset of proportionality constants for the entire population was analyzed to obtain the mean and standard deviation of every body measure. Once again, with the exception of hand length and sitting popliteal height, the ANSUR and Japanese populations are observed to differ in terms of the means and standard deviations of the anthropometric proportionality constants.

Tables 3 and 4 also compare the anthropometric data synthesized in Step II of the methodology with the actual data from ANSUR (Gordon et al., 1989) and AIST (National Institute of Bioscience and Human-Technology, Nippon Shuppan Service, Tokyo, 1996); these comparisons are made at the key percentiles used in the synthesis methodology. The tables are complemented by the plots in Figure 6. The error values calculated for each body dimension are the differences between the actual and synthesized values from the $1^{\text {st }}$ to $99^{\text {th }}$ percentiles. There is a high level of agreement between the actual and synthesized values, even at the lower and upper percentiles. The largest deviation (approximately $14 \mathrm{~mm}$, or 0.55 ") occurs at the $95^{\text {th }}$ percentile for female acromial height. This accuracy is valuable for well-informed design decision-making, since it is the users at the tails of anthropometric 
Table 3: A comparison of ANSUR (ANS) and actual (Act) and synthesized (Syn) female Japanese anthropometry at the key percentiles utilized in their synthesis. Weight is in $\mathrm{kg}$, BMI is unit-less, and all other units are in $\mathrm{mm}$.

\begin{tabular}{|c|c|c|c|c|c|c|c|c|}
\hline & & \multicolumn{5}{|c|}{ Percentiles } & \multirow[t]{2}{*}{ Mean } & \multirow[t]{2}{*}{ S.D. } \\
\hline & & $1^{\text {st }}$ & $5^{\text {th }}$ & $50^{\text {th }}$ & $95^{\text {th }}$ & $99^{\text {th }}$ & & \\
\hline \multirow[t]{3}{*}{ Acromial ht } & ANS & 1199 & 1244 & 1332 & 1430 & 1473 & 1334 & 58 \\
\hline & Act & 1174 & 1204 & 1274 & 1370 & 1391 & 1278 & 48 \\
\hline & Syn & 1175 & 1202 & 1274 & 1356 & 1393 & 1277 & 47 \\
\hline \multirow[t]{3}{*}{ Bideltoid brth } & ANS & 381 & 397 & 431 & 471 & 492 & 433 & 23 \\
\hline & Act & 369 & 377 & 405 & 443 & 451 & 407 & 20 \\
\hline & Syn & 369 & 379 & 405 & 438 & 450 & 406 & 18 \\
\hline \multirow[t]{3}{*}{ Butt-knee lnth } & ANS & 523 & 543 & 587 & 640 & 661 & 589 & 30 \\
\hline & Act & 495 & 511 & 540 & 579 & 595 & 541 & 21 \\
\hline & Syn & 495 & 508 & 539 & 579 & 596 & 541 & 22 \\
\hline \multirow[t]{3}{*}{ Butt-popliteal lnth } & ANS & 422 & 440 & 481 & 528 & 544 & 482 & 27 \\
\hline & Act & 395 & 406 & 439 & 473 & 490 & 439 & 21 \\
\hline & Syn & 395 & 407 & 438 & 475 & 490 & 440 & 21 \\
\hline \multirow[t]{3}{*}{ Hand lnth } & ANS & 159 & 165 & 180 & 197 & 205 & 181 & 10 \\
\hline & Act & 156 & 163 & 174 & 188 & 191 & 174 & 7 \\
\hline & Syn & 156 & 161 & 173 & 186 & 191 & 173 & 8 \\
\hline \multirow[t]{3}{*}{ Hip brth, sit } & ANS & 327 & 343 & 383 & 432 & 453 & 385 & 27 \\
\hline & Act & 316 & 330 & 355 & 391 & 402 & 358 & 19 \\
\hline & Syn & 316 & 327 & 355 & 388 & 402 & 356 & 18 \\
\hline \multirow[t]{3}{*}{ Knee ht, sit } & ANS & 455 & 474 & 515 & 561 & 577 & 515 & 26 \\
\hline & Act & 434 & 447 & 474 & 510 & 524 & 475 & 19 \\
\hline & Syn & 434 & 445 & 474 & 509 & 523 & 475 & 19 \\
\hline \multirow[t]{3}{*}{ Popliteal ht, sit } & ANS & 337 & 352 & 389 & 429 & 445 & 389 & 24 \\
\hline & Act & 354 & 362 & 385 & 421 & 430 & 388 & 17 \\
\hline & Syn & 354 & 363 & 385 & 416 & 430 & 387 & 16 \\
\hline \multirow[t]{3}{*}{ Sitting ht } & ANS & 774 & 795 & 851 & 911 & 934 & 852 & 35 \\
\hline & Act & 819 & 825 & 864 & 918 & 932 & 867 & 27 \\
\hline & Syn & 818 & 831 & 864 & 912 & 932 & 868 & 25 \\
\hline \multirow[t]{3}{*}{ Stature } & ANS & 1486 & 1529 & 1628 & 1737 & 1782 & 1629 & 64 \\
\hline & Act & 1484 & 1512 & 1588 & 1690 & 1726 & 1591 & 52 \\
\hline & Syn & 1483 & 1513 & 1588 & 1685 & 1725 & 1594 & 52 \\
\hline \multirow[t]{3}{*}{ Trochanteric ht } & ANS & 761 & 791 & 860 & 937 & 974 & 862 & 45 \\
\hline & Act & 736 & 756 & 815 & 877 & 902 & 814 & 35 \\
\hline & Syn & 738 & 759 & 814 & 877 & 903 & 816 & 36 \\
\hline \multirow[t]{3}{*}{ Weight } & ANS & 46 & 51 & 63 & 79 & 86 & 620 & 84 \\
\hline & Act & 42 & 45 & 51 & 63 & 69 & 53 & 6 \\
\hline & Syn & 42 & 45 & 51 & 64 & 69 & 53 & 6 \\
\hline \multirow[t]{3}{*}{ BMI } & ANS & 18 & 20 & 24 & 29 & 31 & 24 & 3 \\
\hline & Act & 17 & 18 & 21 & 24 & 26 & 21 & 2 \\
\hline & Syn & 17 & 18 & 21 & 25 & 26 & 21 & 2 \\
\hline
\end{tabular}


Table 4: A comparison of ANSUR (ANS) and actual (Act) and synthesized (Syn) male Japanese anthropometry at the key percentiles utilized in their synthesis. Weight is in $\mathrm{kg}$, BMI is unit-less, and all other units are in $\mathrm{mm}$.

\begin{tabular}{|c|c|c|c|c|c|c|c|c|}
\hline & & \multicolumn{5}{|c|}{ Percentiles } & \multirow[t]{2}{*}{ Mean } & \multirow[t]{2}{*}{ S.D } \\
\hline & & $1^{\text {st }}$ & $5^{\text {th }}$ & $50^{\text {th }}$ & $95^{\text {th }}$ & $99^{\text {th }}$ & & \\
\hline \multirow[t]{3}{*}{ Acromial ht } & ANS & 1299 & 1342 & 1442 & 1545 & 1579 & 1443 & 62 \\
\hline & Act & 1245 & 1291 & 1382 & 1481 & 1510 & 1380 & 57 \\
\hline & Syn & 1246 & 1285 & 1382 & 1472 & 1510 & 1380 & 57 \\
\hline \multirow[t]{3}{*}{ Bideltoid brth } & ANS & 434 & 450 & 491 & 534 & 551 & 492 & 26 \\
\hline & Act & 414 & 423 & 455 & 496 & 517 & 456 & 22 \\
\hline & Syn & 414 & 426 & 455 & 499 & 517 & 458 & 22 \\
\hline \multirow[t]{3}{*}{ Butt-knee lnth } & ANS & 552 & 568 & 615 & 668 & 685 & 616 & 30 \\
\hline & Act & 515 & 532 & 574 & 617 & 627 & 574 & 25 \\
\hline & Syn & 516 & 532 & 573 & 611 & 625 & 572 & 24 \\
\hline \multirow[t]{3}{*}{ Butt-popliteal lnth } & ANS & 442 & 458 & 500 & 546 & 563 & 500 & 27 \\
\hline & Act & 414 & 429 & 466 & 505 & 516 & 465 & 22 \\
\hline & Syn & 414 & 428 & 465 & 501 & 515 & 465 & 22 \\
\hline \multirow[t]{3}{*}{ Hand lnth } & ANS & 172 & 179 & 193 & 210 & 219 & 194 & 10 \\
\hline & Act & 173 & 177 & 191 & 205 & 214 & 191 & 9 \\
\hline & Syn & 172 & 178 & 190 & 206 & 214 & 191 & 9 \\
\hline \multirow[t]{3}{*}{ Hip brth, sit } & ANS & 312 & 329 & 365 & 412 & 429 & 367 & 25 \\
\hline & Act & 306 & 317 & 345 & 377 & 392 & 346 & 19 \\
\hline & Syn & 308 & 318 & 345 & 378 & 391 & 346 & 18 \\
\hline \multirow[t]{3}{*}{ Knee ht, sit } & ANS & 497 & 514 & 557 & 605 & 624 & 559 & 28 \\
\hline & Act & 468 & 482 & 518 & 562 & 580 & 520 & 23 \\
\hline & Syn & 468 & 482 & 517 & 561 & 579 & 519 & 24 \\
\hline \multirow[t]{3}{*}{ Popliteal ht, sit } & ANS & 379 & 395 & 433 & 476 & 494 & 434 & 25 \\
\hline & Act & 372 & 387 & 419 & 453 & 465 & 419 & 20 \\
\hline & Syn & 372 & 385 & 419 & 451 & 464 & 418 & 20 \\
\hline \multirow[t]{3}{*}{ Sitting ht } & ANS & 828 & 855 & 914 & 972 & 991 & 914 & 36 \\
\hline & Act & 847 & 871 & 927 & 983 & 1008 & 926 & 34 \\
\hline & Syn & 849 & 870 & 926 & 984 & 1008 & 927 & 35 \\
\hline \multirow[t]{3}{*}{ Stature } & ANS & 1609 & 1649 & 1756 & 1868 & 1909 & 1756 & 67 \\
\hline & Act & 1570 & 1612 & 1716 & 1818 & 1855 & 1714 & 63 \\
\hline & Syn & 1571 & 1613 & 1715 & 1815 & 1856 & 1714 & 61 \\
\hline \multirow[t]{3}{*}{ Trochanteric ht } & ANS & 825 & 854 & 926 & 1008 & 1039 & 928 & 48 \\
\hline & Act & 791 & 802 & 877 & 941 & 967 & 876 & 41 \\
\hline & Syn & 791 & 816 & 876 & 940 & 966 & 877 & 38 \\
\hline \multirow[t]{3}{*}{ Weight } & ANS & 56 & 63 & 79 & 101 & 109 & 80 & 11 \\
\hline & Act & 48 & 50 & 63 & 76 & 87 & 63 & 8 \\
\hline & Syn & 48 & 52 & 63 & 80 & 87 & 65 & 8 \\
\hline \multirow[t]{3}{*}{ BMI } & ANS & 20 & 21 & 26 & 31 & 34 & 26 & 3 \\
\hline & Act & 17 & 18 & 21 & 25 & 28 & 22 & 2 \\
\hline & Syn & 17 & 18 & 21 & 26 & 28 & 22 & 3 \\
\hline
\end{tabular}


Table 5: A comparison of the mean and standard deviations of the anthropometric proportionality constants calculated for female ANSUR and the actual and synthesized female Japanese populations.

\begin{tabular}{rcccc}
\hline & \multicolumn{2}{c}{ ANSUR } & & \multicolumn{2}{c}{ Japan } \\
\cline { 2 - 3 } \cline { 5 - 5 } & & Mean (Std. dev.) & & Mean (Std. dev.) \\
& & & Actual & Synthesized \\
\hline Acromial ht & $0.818(0.009)$ & & $0.803(0.009)$ & $0.801(0.008)$ \\
Bideltoid brth & $0.266(0.014)$ & & $0.256(0.012)$ & $0.255(0.011)$ \\
Butt-knee lnth & $0.361(0.012)$ & & $0.340(0.008)$ & $0.340(0.009)$ \\
Butt-popliteal lnth & $0.296(0.011)$ & & $0.276(0.008)$ & $0.276(0.009)$ \\
Hand lnth & $0.111(0.005)$ & & $0.109(0.003)$ & $0.109(0.004)$ \\
Hip brth, sit & $0.236(0.016)$ & & $0.225(0.012)$ & $0.224(0.011)$ \\
Knee ht, sit & $0.316(0.008)$ & & $0.299(0.006)$ & $0.298(0.006)$ \\
Popliteal ht, sit & $0.239(0.009)$ & & $0.244(0.006)$ & $0.243(0.006)$ \\
Sitting ht & $0.523(0.015)$ & & $0.545(0.011)$ & $0.545(0.012)$ \\
Trochanteric ht & $0.529(0.015)$ & & $0.512(0.010)$ & $0.512(0.012)$ \\
\hline
\end{tabular}

Table 6: A comparison of the mean and standard deviations of the anthropometric proportionality constants calculated for male ANSUR and the actual and synthesized male Japanese populations.

\begin{tabular}{rcccc}
\hline & \multicolumn{2}{c}{ ANSUR } & & \multicolumn{2}{c}{ Japan } \\
\cline { 2 - 3 } \cline { 5 - 5 } \cline { 5 - 5 } & & Mean (Std. Dev.) & & Mean (Std. dev.) \\
& & & Actual & Synthesized \\
\hline Acromial ht & $0.821(0.010)$ & & $0.805(0.009)$ & $0.805(0.009)$ \\
Bideltoid brth & $0.280(0.014)$ & & $0.266(0.012)$ & $0.267(0.013)$ \\
Butt-knee lnth & $0.351(0.010)$ & & $0.335(0.008)$ & $0.334(0.008)$ \\
Butt-popliteal lnth & $0.285(0.010)$ & & $0.272(0.008)$ & $0.271(0.008)$ \\
Hand lnth & $0.110(0.004)$ & & $0.111(0.004)$ & $0.111(0.004)$ \\
Hip brth, sit & $0.209(0.013)$ & & $0.202(0.010)$ & $0.202(0.010)$ \\
Knee ht, sit & $0.318(0.008)$ & & $0.303(0.007)$ & $0.303(0.007)$ \\
Popliteal ht, sit & $0.247(0.008)$ & & $0.244(0.007)$ & $0.244(0.006)$ \\
Sitting ht & $0.521(0.014)$ & & $0.540(0.011)$ & $0.541(0.014)$ \\
Trochanteric ht & $0.529(0.014)$ & & $0.511(0.011)$ & $0.512(0.011)$ \\
\hline
\end{tabular}


distributions who are at the highest risk of being disaccommodated by the decisions. Greater accuracy in the anthropometric information about these portions of the distributions can help make better-informed, more responsible decisions.

The similarities in the values of actual and synthesized data across a range of percentiles may be insufficient evidence for the accuracy of the proposed methodology. This mode of comparison can verify the accuracy of the data synthesized for each body dimension, but not the effectiveness of the sampling procedure (Step III) to yield realistic and accurate combinations of anthropometry for a target population characterized by different body proportions. The proportionality constants-based comparisons in Tables 5 and 6 are therefore used to supplement the comparisons made through Tables 3 and 4 . The proposed methodology is verified to be capable of replicating a population's unique characteristics as represented by the body proportions. For each population examined, a comparison of the "actual" and "synthesized" summary statistics shows that there is an agreement in the means and standard deviations of the proportionality constants.

As a final test, the PCD-based correlations procedure in Tables 1 and 2 is utilized once more as an additional means of evaluating the PCD approach. The same 28 anthropometry pairs that were identified for the Japan-ANSUR comparison were retained for an examination of the actual and synthesized Japanese populations. Percentage differences were calculated between the PCD-based correlation coefficients for these anthropometry pairs in the actual and synthesized data. The differences were found to be less than $5 \%$ for 21 and 24 anthropometry pairs in the female and male data, respectively (Tables 1 and 2). The correlation coefficients differed by more than $10 \%$ for only 1 and 3 pairs respectively in the female and male data. These findings demonstrate a general correspondence of the inter-anthropometric correlations in the actual and synthesized datasets.

These observations indicate that the proposed methodology is able to synthesize detailed and accurate anthropometric data using information about a target population at only 5 key percentiles (in this case, $1^{\text {st }}, 5^{\text {th }}, 50^{\text {th }}, 95^{\text {th }}$, and $\left.99^{\text {th }}\right)$. The implications of these observations and results on effective design decision-making are discussed in the following section. 


\section{Discussion}

The desired characteristics of the proposed anthropometry synthesis methodology were simplicity, ease of application, applicability using readily available anthropometric information (e.g., values of body measures at different percentiles), and accuracy across varied populations. These attributes of the methodology were tested in the context of two sets of female and male populations with significant differences in anthropometric distributions: Japanese youth and U.S. military.

The characteristics of the methodology can be discussed in comparison with other possible anthropometry synthesis techniques. As mentioned in Section 1, these include proportionality constants and regression-based approaches. The former, while inherently simple and easy to apply, is limited in accuracy by a number of invalid assumptions about body proportions and their variation within and across populations. The latter, while proven to be simple, easy to apply, and accurate, requires the availability of data about the predictor body dimensions (e.g., stature and BMI) for the target population. The method proposed in this paper is concurrently simple, accurate, and easy to apply. Additionally, owing to its requiring information (i.e., values of body measures at different percentiles) that is readily available for numerous user populations, the method can be implemented for a wide range of populations considered in design efforts.

An important advantage of the proposed method is its effectiveness across source and target populations (in this case, ANSUR and Japanese youth) with varied underlying body proportions. This capability is crucial in maintaining accuracy in synthesis across demographic differences such as distributions of race, ethnicity, nationality, etc., all of which have been shown to influence the anthropometric characteristics (e.g., body proportions) of populations.

In its current form, there are certain limitations of the method. The most significant of these is the lack of validation of the universality of percentile combinations datasets that are derived based on comprehensive and representative databases of anthropometry. However, despite the datasets not being conclusively validated for all possible global populations, this study verified their applicability and effectiveness in the context of ANSUR and Japanese youth, which were shown to be two statistically different populations. The Mahalanobis distance comparisons in Section 4 revealed a possible explanation for this success: similarities in underlying statistical patterns across the 
anthropometry of the two populations. Future work will look to test this hypothesis across more populations.

A second limitation is the required size and variability of the target population. The assumption of the universal validity of the ANSUR-derived percentile combination datasets is dependent on the target population being large and varied enough to be approximately represented by the variability captured in the dataset. Certain populations (e.g., beauty pageant contestants) may consist of relatively small numbers of users. Certain other populations (e.g., race horse jockeys) may be characterized by high degrees of anthropometric homogeneity. The anthropometric variability in the universal percentile datasets is unlikely to be applicable for such user groups.

A third potential limitation is the model-fitting procedure described in Section 3.3 and illustrated in Figure 5. The method involved first assuming that the underlying body measure data was approximately Gaussian in nature, and then fitting two separate Gaussian models to the upper and lower halves of the body measure data based on the available anthropometric values at different percentiles. While this yielded satisfactorily accurate anthropometry estimates in this study, the assumption of normality of the underlying data is known to be invalid for certain body dimensions (Vasu \& Mital, 2000). For instance, measures of breadth, circumference, and weight tend to be more lognormal in nature (Brainard \& Burmaster, 1992).

There is therefore scope for the application of improved optimization routines in achieving better fits of the resulting models to the underlying data. However, the model-fitting procedure suggested in this paper has been shown to be satisfactorily accurate in the context of the case study populations. Furthermore, increased levels of complexity could result in the applicability of the procedure being restricted to a smaller, less varied range of populations. In its current form, its inherent simplicity could allow for easy application in numerous and diverse contexts. The development of techniques that are similarly simple and yet more accurate can also be the focus of future research.

A final topic that could be explored more thoroughly is the number of individuals $(\mathrm{m})$ and number of body dimensions $(\mathrm{n})$ that are required for a PCD to capture the anthropometric variability within the underlying population. The PCD must account for the entire range of multidimensional anthropometric variability within a population. The PCD can be utilized to synthesize data for other populations only if it captures the entire extent of variability within the reference population. However, anthropometry varies to different degrees in different populations. For example, ANSUR, which is 
a military population, is more homogeneous than the NHANES databases, which represent civilian populations. Accordingly, for a given reference population, it would be useful to know the minimum value of $\mathrm{m}$ (number of individuals) that would result in a robust PCD of $\mathrm{n}$ body dimensions of interest.

\section{Conclusions}

This work proposed the percentile combinations datasets (PCD) technique to synthesize comprehensive anthropometric databases for populations based on easily-available information about the values of body measures at different percentiles. The method consists of three steps: collecting descriptive statistics about anthropometry, fitting anthropometric models to this information, and sampling the anthropometric models to generate the required data. The application of this procedure was demonstrated in the context of two different populations: the U.S. military in the late 1980s and Japanese youth in the early 1990s. The methodology was shown to be simple, easy to apply, and accurate.

\section{Acknowledgements}

This research was partially funded by the National Science Foundation under Award No. 1131467. Any opinions, findings, and conclusions or recommendations expressed in this material are those of the author(s) and do not necessarily reflect the views of the National Science Foundation.

\section{References}

Annis, J. F. (1996). Aging effects on anthropometric dimensions important to workplace design. International Journal of Industrial Ergonomics, 18, 381-388.

Barroso, M. P., Arezes, P. M., da Costa, L. G., \& Miguel, A. S. (2005). Anthropometric study of portuguese workers. International Journal of Industrial Ergonomics, 35, 401 - 410.

Blackwell, S., Brill, T., Boehmer, M., Fleming, S., Kelly, S., Hoeferlin, D., \& Robinette, K. (2008). CAESAR Survey Measurement and Landmark Descriptions. Technical Report Air Force Research Laboratory, WrightPatterson AFB, OH. 
Brainard, J., \& Burmaster, D. (1992). Bivariate distributions for height and weight of men and women in the United States. Risk Analysis, 12, $267-275$.

Case, K., Porter, M., Gyl, D., Marshall, R., \& Oliver, R. (2001). Virtual fitting trials in 'design for all'. Journal of Materials Processing Technology, $117,255-261$.

Chapman, K. (2011). Sizing up virtual fit technology. www.techexchange.com/library/Sizing\%20up\%20Virtual\%20Fit\%20

Technology.pdf.

Drillis, R., \& Contini, R. (1966). Body segment parameters. New York, cited in ?: Office of Vocational Rehabilitation Engineering \& Science.

Drury, C. (2008). The future of ergonomics/the future of work: 45 years after Bartlett (1962). Ergonomics, 51, 14-20.

Garneau, C., \& Parkinson, M. (2009). Optimization of tool handle shape for a target user population. In Proceedings of the ASME International Design Engineering Technical Conferences DETC2009-87444. San Diego, CA: ASME International.

Garneau, C., \& Parkinson, M. (2010). Visual analysis of user accommodation. In ASME Design Engineering Technical Conferences DETC2010-28712. Montreal, Quebec, Canada: ASME International.

Gite, L., Majumder, J., Mehta, C., \& Khadatkar, A. (2009). Anthropometric and strength data of Indian agricultural workers for farm equipment design. All India Coordinated Research Project on Ergonomics and Safety in Agriculture, Central Institute of Agricultural Engineering, Bhopal, India.

Godwin, A., Eger, T., Salmoni, A., Grenier, S., \& Dunn, P. (2007). Postural implications of obtaining line-of-sight for seated operators of underground mining load-haul-dump vehicles. Ergonomics, 50, 192-207.

Gordon, C. C., Churchill, T., Clauser, C. E., Bradtmiller, B., McConville, J. T., Tebbetts, I., \& Walker, R. A. (1989). 1988 Anthropometric Survey of U.S. Army Personnel: Methods and Summary Statistics, Final Report. 
Technical Report NATICK/TR-89/027 U.S. Army Natick Research, Development and Engineering Center Natick, MA.

Guan, J., Hsiao, H., Bradtmiller, B., Kau, T.-Y., Reed, M. P., Jahns, S. K., Loczi, J., Hardee, H. L., \& Piamonte, D. P. T. (2012). U.S. truck driver anthropometric study and multivariate anthropometric models for cab designs. Human Factors: The Journal of the Human Factors and Ergonomics Society, 54, 849-871.

Hu, H., Li, Z., Yan, J., Wang, X., Xiao, H., Duan, J., \& Zheng, L. (2007). Anthropometric measurement of the Chinese elderly living in the Beijing area. International Journal of Industrial Ergonomics, 37, 303 - 311.

Imrhan, S. N., Nguyen, M.-T., \& Nguyen, N.-N. (1993). Hand anthropometry of Americans of Vietnamese origin. International Journal of Industrial Ergonomics, 12, $281-287$.

Instituto Nacional de Tecnologia (1995). ERGOKIT. Divisao de Desenho Industrial, Instituto Nacional de Tecnologia, Brazil.

ISO (2010). ISO/TR 7250-2:2010. International Organization for Standardization.

Jarosz, E. (1999). Anthropometry of elderly women in poland: dimensions for design. International Journal of Industrial Ergonomics, 25, 203 - 213.

Kee, D., \& Karwowski, W. (2001). The boundaries for joint angles of isocomfort for sitting and standing males based on perceived comfort of static joint postures. Ergonomics, 44, 614-648.

Kolich, M., \& Taboun, S. M. (2004). Ergonomics modelling and evaluation of automobile seat comfort. Ergonomics, 47, 841-863.

Liu, W. C. V., Sanchez-Monroy, D., \& Parga, G. (1999). Anthropometry of female maquiladora workers. International Journal of Industrial Ergonomics, 24, $273-280$.

Mahalanobis, P. (1936). On the generalised distance in statistics. Proceedings of the National Institute of Science, India, 2, 49-55. 
Mohammed, Y. A. A. (2005). Anthropometric characteristics of the hand based on laterality and sex among Jordanian. International Journal of Industrial Ergonomics, 35, 747-754.

Mokdad, M. (2002). Anthropometric study of Algerian farmers. International Journal of Industrial Ergonomics, .

Mokdad, M., \& Al-Ansari, M. (2009). Anthropometrics for the design of bahraini school furniture. International Journal of Industrial Ergonomics, 39, $728-735$.

Moroney, W., \& Smith, M. (1972). Empirical reduction in Potential User Population as the Result of Imposed Multivariate Anthropometric Limits. Technical Report NAMRL-1164 (AD 752 032) Naval Aerospace Medical Research Labratory Report Pensacola, FL.

Nadadur, G., \& Parkinson, M. (2009). Consideration of demographics and variance in regression approaches to estimating body dimensions for spatial analysis of design. ASME Journal of Mechanical Design, 132, 021007.

National Institute of Bioscience and Human-Technology, Nippon Shuppan Service, Tokyo (1996). AIST anthropometric database 1991-1992, Human body dimensions data for ergonomic design.

O'Sullivan, L., \& Gallwey, T. (2005). Forearm torque strengths and discomfort profiles in pronation and supination. Ergonomics, 48, 703-721.

Paquet, V., \& Feathers, D. (2004). An anthropometric study of manual and powered wheelchair users. International Journal of Industrial Ergonomics, 33, 191 - 204. Anthropometry and disability.

Pennathur, A., \& Dowling, W. (2003). Effect of age on functional anthropometry of older mexican american adults: a cross-sectional study. International Journal of Industrial Ergonomics, 32, 39 - 49.

Reed, M. P. (2005). Development of a New Eyellipse and Seating Accommodation Model for Trucks and Buses. Technical Report UMTRI-2005-30 University of Michigan Transportation Research Institute Ann Arbor, MI.

Roebuck, J. A. (1995). Anthropometric methods: designing to fit the human body. HFES. Santa Monica, CA. 
Ruff, C. (2002). Variation in human body size and shape. Annual Review of Anthropology, 31, 211-232.

Strasser, H. (1995). Ergonomic efforts aiming at compatibility in work design for realizing preventive occupational health and safety. International Journal of Industrial Ergonomics, 16, 211-235.

The State Bureau of Technical Supervision (1989). Human dimensions of Chinese adults (GB 10000-88).

Tilley, A. R., \& Dreyfuss, H. (2001). The measure of man and woman: human factors in design. John Wiley \& Sons, Inc.

U.S. Centers for Disease Control and Prevention (2008). National Health and Nutrition Examiniation Survey (NHANES). National Center for Health Statistics.

Vasu, M., \& Mital, A. (2000). Evaluation of the validity of anthropometric design assumptions. International Journal of Industrial Ergonomics, 26, $19-37$.

de Vries, C., Garneau, C., Nadadur, G., \& Parkinson, M. (2011). Considering secular and demographic trends in designing for present and future populations. ASME Journal of Mechanical Design, 133, 081008.

Wang, E., Wang, M., Yeh, W., Shih, Y., \& Lin, Y. (1998). Development of anthropometric work environment for taiwanese workers. International Journal of Industrial Ergonomics, 23, 3-8. 

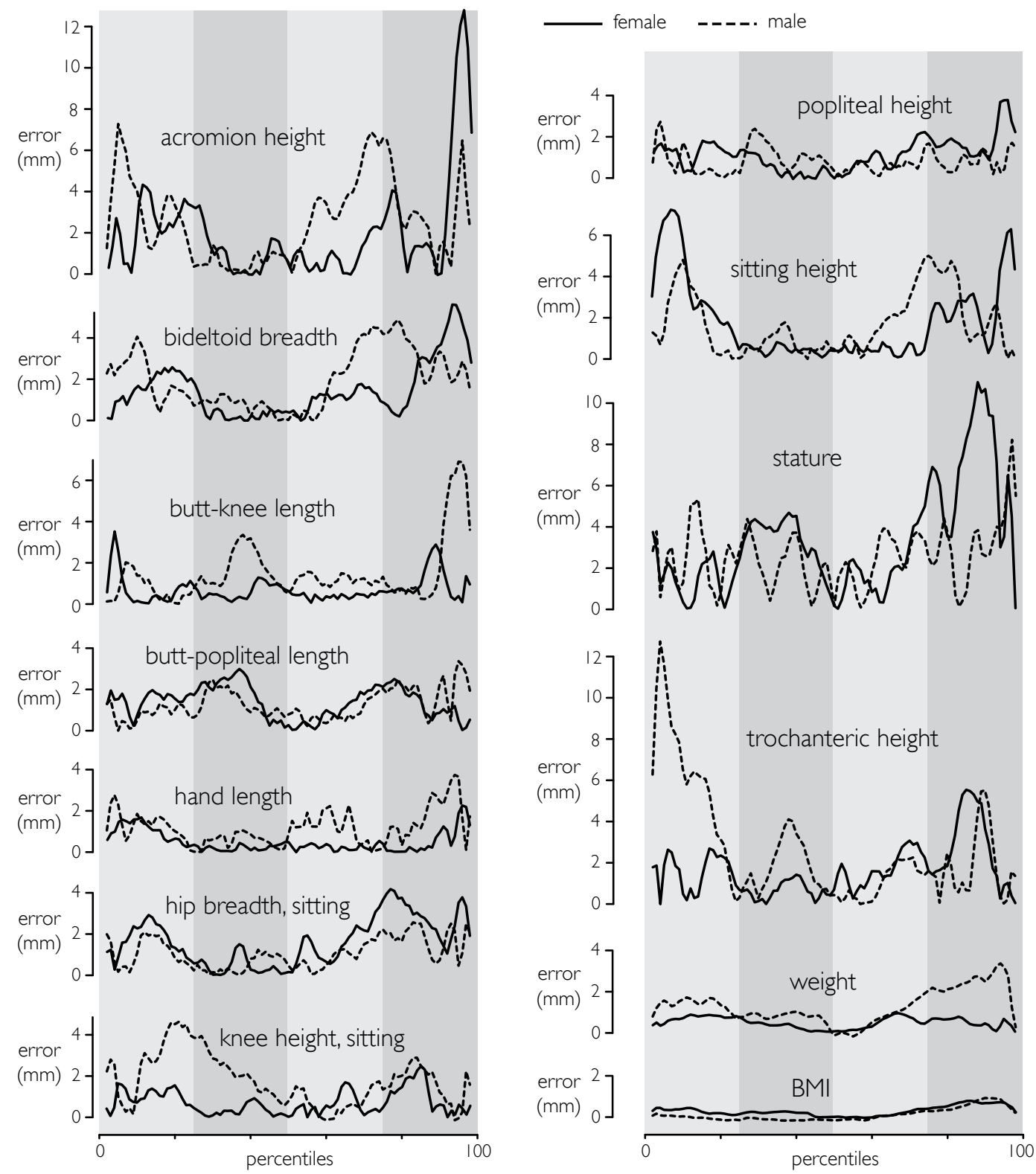

Figure 6: Errors in synthesized anthropometry for the female and male Japan populations. For each body dimension, error at a percentile is the difference between the corresponding values of actual and synthesized anthropometry. 